\section{Oral contraceptives and cancer}

Many of this Journal's older readers will be familiar with the Oxford-Family Planning Association (Oxford-FPA) contraceptive study and will, indeed, have made important contributions to data collection. Accordingly, I was delighted that the Journal Editor had chosen the most recent publication from the study ${ }^{1}$ as the subject for a Journal Review. I am also grateful to Dr Mills for taking so much trouble to produce a succinct summary of a complex paper. ${ }^{2}$ There are, however, one or two points about the review to which I would like to draw readers' attention.

First, I would like to stress that the majority of the women in the study were followed up individually until mid-1994, although individual follow-up for a substantial subgroup of women ceased earlier than this. With regard to cancer registrations and death notifications, all women (save for those who emigrated) were followed up until the end of 2004 using information provided by the National Health Service Central Registries to supplement data collected during the course of individual follow-up

Second, the Journal Review does not include any confidence intervals for the rate ratios (no doubt in the interests of saving space). This is, perhaps, of concern mainly for invasive cervical cancer where the Oxford-FPA findings were considerably more unfavourable than has been described in most other studies. As the Oxford-FPA study included only 59 cases of this disease (with only six cases in the reference group who never used oral contraceptives), confidence intervals around the rate ratios were wide.

Finally, while the population studied was certainly of higher social class than the general population, it was not "predominantly Social Class I". The paper only gives the proportion of women from Social Classes I and II combined and this figure was $41 \%$.

These are relatively minor points that do not detract from the substance and conclusions of the careful review prepared by Dr Mills.

Martin Vessey, CBE, FRS

Emeritus Professor of Public Health,

University of Oxford, Oxford, UK.

E-mail: martin.vessey@dphpc.ox.ac.uk

References

Vessey M, Painter R. Oral contraceptive use and cancer: findings in a large cohort study, 1968-2004. Br J Cancer 2006; 95: 385-389.

2 Mills P. Journal review of 'Oral contraceptive use and cancer: findings in a large cohort study, 1968-200

\section{Increase in IUD expulsions}

Further to the letters of Drs Hawkins and Callander ${ }^{1}$ and my own, ${ }^{2,3}$ I received a telephone call from a doctor in Hong Kong expressing satisfaction of changing practice of fitting and found entirely satisfactory since 1996 .

He tried hard to find a manufacturer to adopt the new design and found one in India. He fits 100 intrauterine devices a month with no problems. He drew my attention to an article 4 that supports my proposal, the abstract of which is as follows:

Objective: To assess the validity of modifying the technique of intrauterine device (IUD) placement to decrease the incidence of incorrect positioning within the uterine cavity. Methods: We used the current applicator in 78 women and an applicator shortened by $1.5 \mathrm{~cm}$ in 91 women and examined the uterus by vaginal ultrasonography before and after application in both groups. Results: Six of the 78 unmodified insertions were found to be incorrectly placed, while none of the IUDs inserted with the new technique was placed incorrectly. Conclusions: We suggest shortening the applicator or lengthening the push rod to increase the likelihood of proper IUD insertion and thereby enhance performance.

Rajendra Prasad Yadava, FRCGP, FFFP General Practitioner and Instructing Doctor, Merton Surgery, Longton, Stoke-on-Trent, UK

E-mail: rajendra.yadava@nhsawebmail.nhs.uk

References

Hawkins F Callander F Increase in IUD expulsions (Letter). J'Fam Plann Reprod Health Care 2006; 32 : Yadava RP Ortho Gynae T380 Slimline fitting made easier? (Letter). Br J Fam Plann 1995; 21: 78. Yadava RP. Increase in IUD expulsions Plann Reprod Health Care 2007; 33: 65-66.

Homouda K. The effect on intrauterine device position and performance of a modified TCu380A insertion echnique. Eur J Contracept Reprod Health Care 2002; 7: 31-35.

\section{IUD expulsions}

I would like to express my total agreement with Dr Yadava in his letter entitled 'Increase in IUD expulsions' published in the January 2007 issue of the Journal.

It was some years ago that I noted the rod in the T-Safe 380A ${ }^{\circledR}$ intrauterine device was about $1 \mathrm{~cm}$ shorter than the introducer tube. Since this discovery, and the feeling that the device seemed to 'cling' to the tube while the latter was being removed, I have systematically shortened the tube before insertion in exactly the same way as Dr Yadava describes, and have explained to others the reason why.

I cannot prove that the expulsion rate of these 'adjusted' fittings has fallen, but the fitting procedure is a more satisfying experience.

\section{Margaret Curtis, FFFP}

Semi-retired Consultant in Community Family Planning, Aylesbury, UK

Reference

Yadava RP. Increase in IUD expulsions (Letter). J Fam Plann Reprod Health Care 2007; 33: 65-66.

\section{Pressed for time: providing the} Standard Days Method and oral contraceptives in India

Recently, we reported in this Journal ${ }^{1}$ that Rwandan providers exchanged on average 39 items of relevant information as they counselled clients who chose the Standard Days Method (SDM) ${ }^{2}$ and only 31 with those who chose pills. Sixteen specific SDM items and only six pill items were addressed by at least $95 \%$ of providers in the consultations. We concluded that SDM counselling is generally satisfactory and that providers perform below capacity when they deliver counselling on pills. These findings emerged in clinics of the Ministry of Health $(\mathrm{MOH})$ in which providers offered consultations that lasted about 44 minutes with SDM clients and 38 minutes with pill clients

Yet, in many settings, practical constraints limit the amount of time a family planning provider can spend with a client, which may impair the quality of care. Frequently, client load is overwhelming and time becomes a scarce asset: providers must see as many clients as possible each day. At $\mathrm{MOH}$ clinics of the Ranchi district in the Jharkhand state of India, providers engage in short consultations. To test the generalisation of the Rwandan findings, we replicated the study in 69 clinics of the Kanke and Ormanjhi blocks of Ranchi. The SDM was introduced through a 2-day workshop for providers after a 2-day contraceptive update workshop that reinforced pill (and other methods) delivery.

The consultations lasted on average 9 minutes and 41 seconds with SDM clients and 8 minutes and 4 seconds with pill clients. The number of information items exchanged with SDM clients reached 23.20 on average and with pill clients 21.75 . Only 16 specific items from the SDM checklist were addressed by at least $60 \%$ of providers. Essential contraindication questions (e.g. Asked whether my periods come more or less when I expect them, 59\%; Asked about partner's willingness to abstain or use protection on fertile days, $48 \%$ ) and follow-up instructions (e.g. Told me to see the provider if period does not return the day after the band passes over the last bead, $46 \%$; See the provider if period returns before the day on which the band should reach the dark brown bead, $43 \%$ ) routinely failed to meet the $60 \%$ standard. Important gaps were found in pill counselling as well (e.g. Asked about my blood pressure or measured it, or someone else did, 3\%; Told me to return to the clinic right away if I have severe headaches and/or blurry vision, 9\%; To return if I have any question or concern, $38 \%$ )

Nonetheless, the study results suggest that SDM and pills might be successfully offered in less than 10 minutes if counselling were further streamlined and focused. This will require prioritising items in the service delivery protocol and training providers to focus counselling on essential topics. A satisfactory list of 23 essential SDM items can be established and emphasised in SDM training. If providers, instead of using a more personal selection of items from the extensive SDM protocol, addressed such 23 items in 9 minute and 41 second consultations, they would cover all of the basic topics of SDM counselling despite the limited session length. Analogous recommendations apply to pill counselling.

The SDM and pill results from our India study suggest that counselling training for providers pressed for time must help them select from the extensive information contained in method delivery protocols, family planning technology tables, or national reproductive health care guidelines a subset of essential items for standard use in interactions with clients.

Federico León, PhD

Consultant, Georgetown University, Institute for Reproductive Health, Ate, Lima, Peru.

E-mail:frleon@amauta.rcp.net.pe

Rebecka Lundgren, MA

Senior Researcher, Georgetown University, Institute for Reproductive Health, Washington, $D C, U S A$

Ana Huapaya, BSc

Statistician, Instituto para la Salud

Reproductiva, Miraflores, Lima, Peru

Priya Jha, MA

Researcher, Georgetown University, Institute for Reproductive Health - India Field Office, New Delhi, India

Ragini Sinha, $\mathrm{MA}$

Researcher, Krishi Gram Vikas Kendra, c/o Usha Martin Industries Ltd, Ranchi, Jharkhand, India

Victoria Jennings, $\mathrm{PhD}$

Director, Georgetown University, Institute for Reproductive Health, Washington, DC, USA

\section{Reference}

León FR, Blair C, Huapaya A, Lundgren R, Mukabatsinda M, Muramutsa F, Jennings V. Quality of delivery of the Standard Days Method as compared with contraceptive pills in Rwanda. J Fam Plann Reprod Health Care 2006; 32: 231-233.

2 Gribble JN, Jennings V, Nikula M. Mind the gap: responding to the global funding crisis in family planning. J Fam Plann Reprod Health Care 2004; 30: 155-157. 